\title{
Presurgical weight loss affects tumour traits and circulating biomarkers in men with prostate cancer
}

Wendy Demark-Wahnefried ${ }^{\star, 1,2}$, Soroush Rais-Bahrami ${ }^{2,3,4}$, Renee A Desmond ${ }^{2,5}$, Jennifer B Gordetsky ${ }^{3,6}$, Gary R Hunter ${ }^{7}$, Eddy S Yang ${ }^{2,8}$, Maria Azrad ${ }^{1}$, Andrew D Frugé ${ }^{1}$, Yuko Tsuruta ${ }^{1}$, Lyse A Norian ${ }^{1,2}$, Roanne Segal ${ }^{9}$ and William E Grizzle ${ }^{2,6}$

${ }^{1}$ Department of Nutrition Sciences, Wallace Tumor Institute, University of Alabama at Birmingham, 1824 6th Avenue South, Suite 102, Birmingham, AL 35294, USA; ${ }^{2}$ University of Alabama at Birmingham Comprehensive Cancer Center, Wallace Tumor Institute, 1824 6th Avenue South, Birmingham, AL 35294, USA; ${ }^{3}$ Department of Urology, University of Alabama at Birmingham, 619 19th Street South, Birmingham, AL 35233, USA; ${ }^{4}$ Department of Radiology, 619 19th Street South, Birmingham, AL 35233, USA; ${ }^{5}$ Division of Preventive Medicine, University of Alabama at Birmingham, 1717 11th Avenue South, Birmingham, AL 35205, USA; ${ }^{6}$ Division of Anatomic Pathology, Department of Pathology, University of Alabama at Birmingham, 619 19th Street South, Birmingham, AL 35233, USA; 'Department of Human Studies, University of Alabama at Birmingham, 901 13th Street South, Birmingham, AL 35294, USA; ${ }^{8}$ Department of Radiation Oncology, University of Alabama at Birmingham, 1700 6th Avenue South, Birmingham, AL 35233, USA and ${ }^{9}$ Department of Medicine, University of Ottawa, 1053 Carling Avenue, Ottawa, ON K1Y 4E9, Canada

Background: Obesity is associated with aggressive prostate cancer. To explore whether weight loss favourably affects tumour biology and other outcomes, we undertook a presurgical trial among overweight and obese men with prostate cancer.

Methods: This single-blinded, two-arm randomised controlled trial explored outcomes of a presurgical weight loss intervention (WLI) that promoted $\sim 1 \mathrm{~kg}$ per week loss via caloric restriction and increased physical activity (PA). Forty overweight/obese men with clinically confirmed prostate cancer were randomised to the WLI presurgery or to a control arm; changes in weight, body composition, quality-oflife, circulating biomarkers, gene expression, and immunohistochemical markers in tumour and benign prostatic tissue were evaluated.

Results: The study period averaged 50 days. Mean (s.d.) change scores for the WLI vs control arms were as follows: weight: -4.7 (3.1) kg vs $-2.2(4.4) \mathrm{kg}(P=0.0508)$; caloric intake: -500 (636) vs -159 (600) kcal per day $(P=0.0034)$; PA: $+0.9(3.1)$ vs $+1.7(4.6)$ MET-hours per day (NS); vitality: $+5.3(7.14)$ vs $-1.8(8.1)(P=0.0491)$; testosterone: +55.1 (86.0) vs $-48.3(203.7) \mathrm{ng} \mathrm{dl}^{-1}$ $(P=0.0418)$; sex hormone-binding globulin: $+14.0(14.6) v s+1.8(7.6) \mathrm{nmoll} \mathrm{I}^{-1}(P=0.0023)$; and leptin: $-2.16(2.6)$ vs $-0.03(3.75)$ $(P=0.0355)$. Follow-up Ki67 was significantly higher in WLI vs control arms; median (interquartile range): $5.0(2.5,10.0)$ vs $0.0(0.0,2.5)$ $(P=0.0061)$ and several genes were upregulated, for example, CTSL, GSK3B, MED12, and LAMC2.

Conclusions: Intentional weight loss shows mixed effects on circulating biomarkers, tumour gene expression, and proliferative markers. More study is needed before recommending weight loss, in particular rapid weight loss, among men with prostate cancer.

A recent consensus report indicates that obesity is a risk factor for 13 different cancers, with accumulating evidence that it may have a role in fatal prostate cancer (Lauby-Secretan et al, 2016). Contributing to this evidence is a multinational study of 10106 prostate cancer cases from 8 different cohorts, which found that for each 5-unit increase in body mass index (BMI: $\mathrm{kg} \mathrm{m}^{-2}$ ) pre-diagnosis, there was an $8 \%$ increase in prostate cancer specific mortality $(P$-trend $=0.01)$ (Yuan et al, 2015). A meta-analysis among 26479 prostate cancer patients 
found a $21 \%$ increased risk of biochemical recurrence with a similar increase in weight (relative risk: $1.21,95 \%$ confidence interval: $1.11-$ 1.31, $P<0.01$ ) (Cao and Ma, 2011). Despite strong observational evidence that obesity is associated with more aggressive prostate cancer, few studies have explored whether intentional weight loss results in improved cancer control and/or possible mechanisms by which negative energy balance affects tumour biology and the host environment. Through such research, it may be possible to discover new supportive therapies and uncover signalling pathways that lead to the development of novel therapeutic agents (Hursting et al, 2012).

Limited research in breast cancer shows that weight loss interventions (WLIs) are safe for cancer survivors and improve health-related quality-of-life in the short-term (Reeves et al, 2014). A limited number of trials also suggest improvements in circulating inflammatory markers and adipokines, insulin, insulin-like growth factors, sex steroid hormones, and associated binding proteins (Reeves et al, 2014).

In prostate cancer only three randomised controlled trials (RCTs) of weight loss have been reported. RENEW (Reach Out to ENhancE Wellness in Older Cancer Survivors) is the largest and enrolled 261 prostate cancer survivors within a cohort that also included 380 breast and colorectal cancer survivors (Morey et al, 2009). In RENEW, significant reductions in body weight occurred and were associated with improved physical function (primary endpoint). As RENEW did not collect biospecimens, specific physiologic effects were not assessed. Two smaller weight loss trials $(n \leqslant 19)$ (Schenk et al, 2009; Wright et al, 2013) were conducted in the presurgical setting. Here, significant weight loss was observed along with pre-post changes in serum insulin-like growth factor-binding protein-3 between the intervention and control arms; however, no differences were detected in insulin, C-peptide, IGF-1, and adiponectin (Wright et al, 2013). Neither of these trials assessed effects on tumour pathology.

Assessment of the impact of interventions directly on tumour tissue is a strength of presurgical trials-one that Kelloff et al (1994) initially proposed for testing chemopreventive agents. A key premise is that by monitoring intervention effects on Ki67, efficacy could be assessed more rapidly and with fewer participants (Kachroo and Gnanapragasam, 2013). The presurgical model also affords the opportunity to directly assess the impact of intervention on other biological mechanisms within the tumour. Presurgical models have been used to evaluate many therapeutic agents (Dowsett and Dunbier, 2008; Bonanni et al, 2012), but have rarely been used to assess the impact of lifestyle interventions. A phase II, RCT among 161 patients scheduled for prostatectomy, however, found significantly lower Ki67 proliferation rates in men randomised to a 3 -week regimen of $30 \mathrm{~g}$ per day of ground flaxseed $v s$ controls (Demark-Wahnefried et al, 2008), thus serving as proof-of-concept that non-pharmacologic interventions can be tested via presurgical trials. To date, there have been no presurgical trials that have assessed the impact of caloric restriction or increased physical activity (PA) on tumour biology. This report describes the results of a pioneering NCI-funded (R21 CA161263) feasibility trial that utilised a presurgical model to assess the effects of a WLI on tumour biology (Ki67 and gene expression), candidate biomarkers (e.g., circulating prostate specific antigen (PSA), androgens, growth hormones (vascular endothelial growth factor, VEGF), cytokines (tumour necrosis factor (TNF)- $\beta$ ), adipokines (leptin), insulin, and RNA gene expression), and quality-of-life in men with newly diagnosed prostate cancer, who elected radical prostatectomy.

\section{MATERIALS AND METHODS}

Methods of this presurgical RCT are published and can be readily accessed for details (Demark-Wahnefried et al, 2015). A brief summary follows.
Patients. Men aged $\geqslant 19$ years diagnosed with biopsy-confirmed prostate cancer, BMI of $25-50 \mathrm{~kg} \mathrm{~m}^{-2}$, and scheduled for radical prostatectomy $>3$ weeks from the point of contact at the University of Alabama at Birmingham (UAB) or the Urology Centers of Alabama were eligible. Men were excluded if they were unable to communicate in English or via telephone; had other active malignancies (exception: non-melanoma skin cancers) or health conditions that affected body weight, or precluded unsupervised PA (e.g., thyroid dysregulation, severe orthopedic conditions, or unstable/recent cardiovascular issues); had previous hormonal or neo-adjuvant treatment; were unwilling to be randomised; or were currently enrolled in a weight loss programme (note: exclusion was not based on PA level given the focus on short-term weight loss, for which caloric intake has a larger role (Jensen et al, 2014)).

Written informed consent was obtained from all interested and eligible men. The protocol was approved by the UAB Institutional Review Board and registered according to Consolidated Standards of Reporting Trials guidelines (NCT01886677). Recruitment began 7 December 2012 and the final participant completed the protocol 12 February 2015 and tumour specimens were released 1 year thereafter.

Study design. This two-arm, single-blinded, presurgical RCT was conducted among 40 overweight or obese men, newly diagnosed with prostate cancer. The experimental arm was assigned immediately to an energy-restricted diet plus aerobic PA to promote a weight loss of $\sim 1 \mathrm{~kg}$ per week; the wait-list control arm was offered the intervention post surgery.

Objectives and assessments. Designed as a feasibility trial, the primary aims were attainment of the following benchmarks: (1) enrolment of 40 participants within 2 years; (2) $\geqslant 80 \%$ retention; (3) adherence, defined as completion $\geqslant 70 \%$ of contact sessions; and (4) safety, defined as the absence of serious adverse events in the intervention arm. Secondary (exploratory) aims included characterising pre-post between-arm differences on measures of body habitus and composition, energy intake, PA, physical functioning, quality-of-life, serum biomarkers, lymphocytic gene expression, and tumour markers. Phlebotomy and assessments were performed after a $12 \mathrm{~h}$ fast at baseline and follow-up (within 3 days before prostatectomy). Participants exhibiting uncontrolled Stage III hypertension or cardiac abnormality at baseline were cleared by their urologist before study continuation (Schmitz et al, 2010).

\section{Measures/measurement points}

Clinical measures. Anthropometric measures were conducted in light clothing, without shoes, and using standardised procedures (Lohman and Martorell, 1988). Body fat and lean mass were quantified via dual energy X-ray absorptiometry using a calibrated Lunar Prodigy densitometer (GE-Lunar Corporation, Madison, WI; software version 12.3). $\mathrm{VO}_{2}$ assessments were performed using a standardised American College of Sports Medicine (ACSM) protocol in which workout intensity was increased in $4 \mathrm{~min}$ intervals to achieve $80 \%$ of maximum heart rate (MHR) (Kohl et al, 1990). Interval and total treadmill time was recorded. Protocol details are published in the methods paper (DemarkWahnefried et al, 2015); the $\mathrm{VO}_{2 \text { peak }}$ equation is included in Supplementary Materials.

Patient-reported outcomes. Written surveys were administered at baseline and follow-up, to assess all prescribed and over-thecounter medications (including dietary supplements) and comorbidities (Older Americans Resources and Services Comorbidity Index) (Fillenbaum, 1988). Quality-of-life was assessed using the RAND-36, which generated summary scores for Physical Health 
(from physical functioning, physical role limitations, pain, and general health subscales), and Mental Health (from emotional wellbeing, emotional role limitations, vitality, and social functioning subscales); quality-of-life related to urinary, bowel, and sexual functioning was captured using the Prostate Cancer Index) (Hays et al, 1993; Litwin et al, 1998). Physical Activity Recalls (7-Day) were administered by trained personnel (Blair et al, 1985). As indicated in the methods paper, (Demark-Wahnefried et al, 2015) objective PA data were captured using Polar RS400 heart rate monitors (Polar Electro, Inc., Lake Success, NY, USA) (equations are included in Supplementary Materials). Two-day dietary recalls were performed by registered dietitians at both time points (De Keyzer et al, 2011) and analysed for kilocalories, macronutrients, and diet quality using the NCI-developed ASA24 (Subar et al, 2012). All study participants were monitored semi-weekly for adverse events, although none occurred.

Circulating biomarkers. Blood was collected by venipuncture and separated into sera, plasma, neutrophils, leukocytes, and DNA, which were stored at $-80^{\circ} \mathrm{C}$; RNA was dispersed in $0.5 \mathrm{ml}$ of RNAlater and stored at $-20^{\circ} \mathrm{C}$. All sera was batch-analysed in duplicate for leptin, insulin, glucose, total testosterone, sex hormone binding globulin (SHBG), PSA, TNF- $\beta$, and VEGF according to the manufacturer's directions. Details can be accessed in the methods paper, (Demark-Wahnefried et al, 2015) along with a specific description of gene expression profiling for phosphatase and tensin homologue, phosphatidylinositol 3 kinase (PI3K), MAPK, STAT, BCL2, and receptors for insulin, leptin, androgen (AR), VEGF (VEGFR), and TNF (FAS) on circulating RNA extracted from buffy coat.

Tumour biomarkers. Two pathologists, each blinded to study condition, assisted with this investigation. One reviewed clinical pathology reports and all slides for each case, selecting one slide and one block/case based on the presence of adequate tumour, and the representativeness of the specimen; the other confirmed tumour grade. Slides were prepared at a 1:100 dilution for Ki-67 (clone:SP6, Thermo Fisher Scientific, Pittsburg, PA, USA) and percent of positive cells were assessed. Slides for other tumour markers used a 1:20 dilution for 4E-BP1 (clone:11G12C11, Santa Cruz Biotechnology, Dallas, TX, USA), AR (clone: EPR1535(2), Abcam, Cambridge, MA, USA) and IR (Abcam); a 1:200 dilution for cleaved caspase-3 (Asp175, Cell Signaling Technology, Danvers, MA, USA); and a 1:2000 dilution for nuclear factor- $\kappa$-light-chainenhancer of activated B (clone: F-6, Santa Cruz Biotechnology). Intensity levels $(0,1,2,3)$ were multiplied by the percentage staining and divided by 100 .

The NanoString nCounter system (Seattle, WA, USA) was utilised to explore genes involved with signalling and immune pathways within the tumour; this analysis was added post hoc (Demark-Wahnefried et al, 2015). Six cases from each study arm having adequate surgical specimen tumours were selected for analysis; cases from the intervention arm, who lost over the median amount of weight were matched on International Society of Urological Pathology grade, race, and age ( \pm 5 years) to controls with stable weights. Tumour was identified and macrodissected from paraffin-embedded surgical specimens. RNA was isolated using RNeasy FFPE kits (Qiagen, Valencia, CA, USA). Samples were processed according to manufacturer's directions on the NanoString nCounter Flex system using the GX PanCancer Pathways Panel (770 genes representing 13 canonical cancer pathways: 606 pathway genes, 124 cancer driver genes, and 40 reference genes) and the GX PanCancer Immune Profiling Panel (770 genes for 24 immune cell types/populations, 30 common cancer antigens, and genes for overall immune response including key checkpoint blockade genes). All signals below the mean background plus 2 s.d. were considered below the limits of detection. A normalisation factor was calculated from the expression of well-characterised housekeeping genes and spikedin exogenous positive controls in each sample and applied to raw counts from nCounterTM output. The Benjamini-Yekutieli (2001) false discovery rate correction at $P<0.05$ was used, as genes are coregulated within a handful of specific pathways.

Interventions. After baseline assessment, men were block-randomised on race (African American $v s$ others) and baseline BMI $(25-29.9$ vs $30+)$ to receive the WLI immediately or to a wait-list control arm that was offered the weight loss regimen after study completion (Jayachandran et al, 2009; Hadziabdic et al, 2015).

Weight loss intervention arm. The intervention followed American Heart Association, American College of Cardiology, and The Obesity Society guidelines for weight loss, which recommends a tripartite approach of caloric restriction, increased PA, and behaviour modification (Jensen et al, 2014). The Mifflin-St Jeor equation (Frankenfield et al, 2003) was used to calculate energy needs with subtraction of $1000 \mathrm{kcal}$ per day to promote an average weight loss of $\sim 1 \mathrm{~kg}$ per week. Registered dietitians counselled participants on caloric goals and correcting nutrient deficiencies noted from 2-day dietary recalls via food sources. American Cancer Society dietary guidelines were followed (Rock et al, 2012). Dietary instructions were customised to patients' needs and preferences. Participants were provided with references and instructed to count calories or use exchange lists (American Diabetes Association 2007). Participants were instructed to weigh themselves daily; those not having a scale were provided one. Dietitian follow-up occurred semi-weekly with counselling provided face-to-face, via the telephone, or through email and in accordance with Social Cognitive Theory (Bandura, 2004).

Exercise physiologists provided instruction to incrementally and ultimately strive for an additional $250 \mathrm{kcal}$ per day deficit through aerobic PA. Although a reduction in caloric intake is essential to weight loss regimens, guidelines suggest that regular PA also is beneficial to preserve lean mass and provide an additional energy sink to compensate for brief bouts of dietary non-adherence. Consistent with ACSM guidelines, each training session included a 5 min warm-up, the work-out, and then a cool-down of slow walking and stretching (Kohl et al, 1990). An incremental ramping of intensity and volume from 60 to $80 \%$ of MHR as per tolerance was employed. Given that few men obtained regular PA, starting workouts began at $10 \mathrm{~min}$ bouts and increased incrementally, ultimately striving for daily $30 \mathrm{~min}$ sessions. Participants were encouraged to exercise at $\mathrm{UAB}$ twice weekly on ergometers and treadmills, and five times per week at home. Heart rate monitors and instructions were provided to enhance and monitor adherence during home-based sessions. In cases where on-site exercise was not possible due to distance, regimens were adapted to semi-weekly telephone counselling and/or email exchanges based on downloading of heart rate monitor data.

Wait-listed control arm. Wait-listed men also were counseled weekly by dietitians on food sources to correct nutritional deficiencies identified from baseline $24 \mathrm{~h}$ recalls. Wait-listed men were offered a 6-week weight loss regimen post surgery.

\section{Statistical considerations and analyses}

Sample size and statistical power. Power calculations were based on weight loss data from older men (age $50+$ years) enrolled in $\mathrm{UAB}$ weight loss programmes, that is, mean (s.d.) weight loss of 5.64 (3.13) kg over 10 weeks. An enrolment of 40 (20 per arm) with an assumption of $80 \%$ retention yields $98 \%$ power to detect a difference in means of 4.73 (Group 1 mean, $\mu_{1}$ of -5.64 per Group 2 mean, $\mu_{2}$ of -0.91$)$ assuming equal variance and using a two-group, two-sided $t$-test $(\alpha=0.05)$. 
Statistical comparisons. Between-arm differences at baseline were assessed by $t$-tests for continuous variables and Fisher's exact test or $\chi^{2}$-test for categorical variables. Changes in anthropometric measures were computed by subtracting follow-up from baseline values. Between-arm changes were compared using generalised linear models with the change score serving as the dependent variable and the treatment group as a predictor, controlling for baseline measures of pretest scores. Similar models were used for dietary data. Leptin levels were not normally distributed and were log-transformed prior to analyses. Median baseline PSA levels were compared using Wilcoxon tests, as were Ki67 data; for non-zero Ki67 values of $<5$, a value of 2.5 was used. For other immunohistochemical markers comparisons between treatment and control groups at surgery were examined by $t$-tests. Regression models were used to assess relationships between changes in weight, body composition, and serum biomarkers with tumour proliferation rates. Statistical significance was predetermined at $\alpha=0.05$. Nanostring data were analysed using the nSolver software; $P$-values and fold change values were calculated using nCounter default settings that take into consideration background signals measured in negative controls and a normalisation factor described above. Given the exploratory nature of this study, no adjustments were made for multiple comparisons.

\section{RESULTS}

This trial achieved $85 \%$ retention, 95\% adherence, and documented no serious adverse events; the accrual target also was achieved but required six additional months (Demark-Wahnefried et al, 2015). The CONSORT diagram (Figure 1) shows that of the 101 men referred, 40 were enrolled and 34 completed the trial. Other than refusal, prostatectomy-related issues were leading reasons for ineligibility and cancellation of prostatectomy was the leading cause of attrition (four out of six dropouts). One patient withdrew consent upon notification of assignment to the control arm; another was discontinued upon subsequent determination of metastatic disease. No differences were observed between enrollees $v s$ non-enrollees or completers vs non-completers by age, race, or BMI.

Participant characteristics are provided in Table 1. The sample was comprised largely of middle-aged men who were representative of males in the southeastern US in terms of race and education (US Bureau of the Census, 2014). Most participants had moderately aggressive prostate cancer and reported at least three other comorbidities, with roughly half reporting cardiovascular disease and a quarter reporting diabetes. The sample was evenly divided between overweight and obese men. The average length of time on study was 50 days. No statistically significant differences were detected between study arms regarding any of these factors.

Table 2 documents baseline-to-follow-up differences by study arm in body weight and composition, dietary intake, PA, function and fitness, and quality-of-life. Both study arms lost appreciable amounts of weight over the brief study period, with a weight loss of $5 \%$ noted in the WLI arm and $2.2 \%$ in the control arm. Betweenarm differences in weight and BMI over the study period reached borderline significance. Lean and fat mass decreased in both groups with no between-arm differences detected. A significant correlation was observed between the rate of weight loss and loss in lean mass, $\rho=0.632(P=0.0001)$.

Although baseline caloric intakes were lower than expected, possibly due to increased stress with a recent diagnosis, underreporting, or heightened awareness of dietary intake occurring with enrolment in a weight loss trial, caloric intake decreased significantly more over the study period in the WLI arm compared with controls. Significant reductions in intakes across all major macronutrient groups were also observed; however, the relative proportion of carbohydrate, protein, and fat remained stable and did not differ between arms. Slight decreases over time in diet quality were noted in both groups - an effect attributed to decreased dietary variety concomitant with decreased overall intakes.

Both arms also reported modest increases in weekly minutes of moderate-to-vigorous PA, although no between-arm differences were observed and the net impact on weekly Metabolic Equivalent

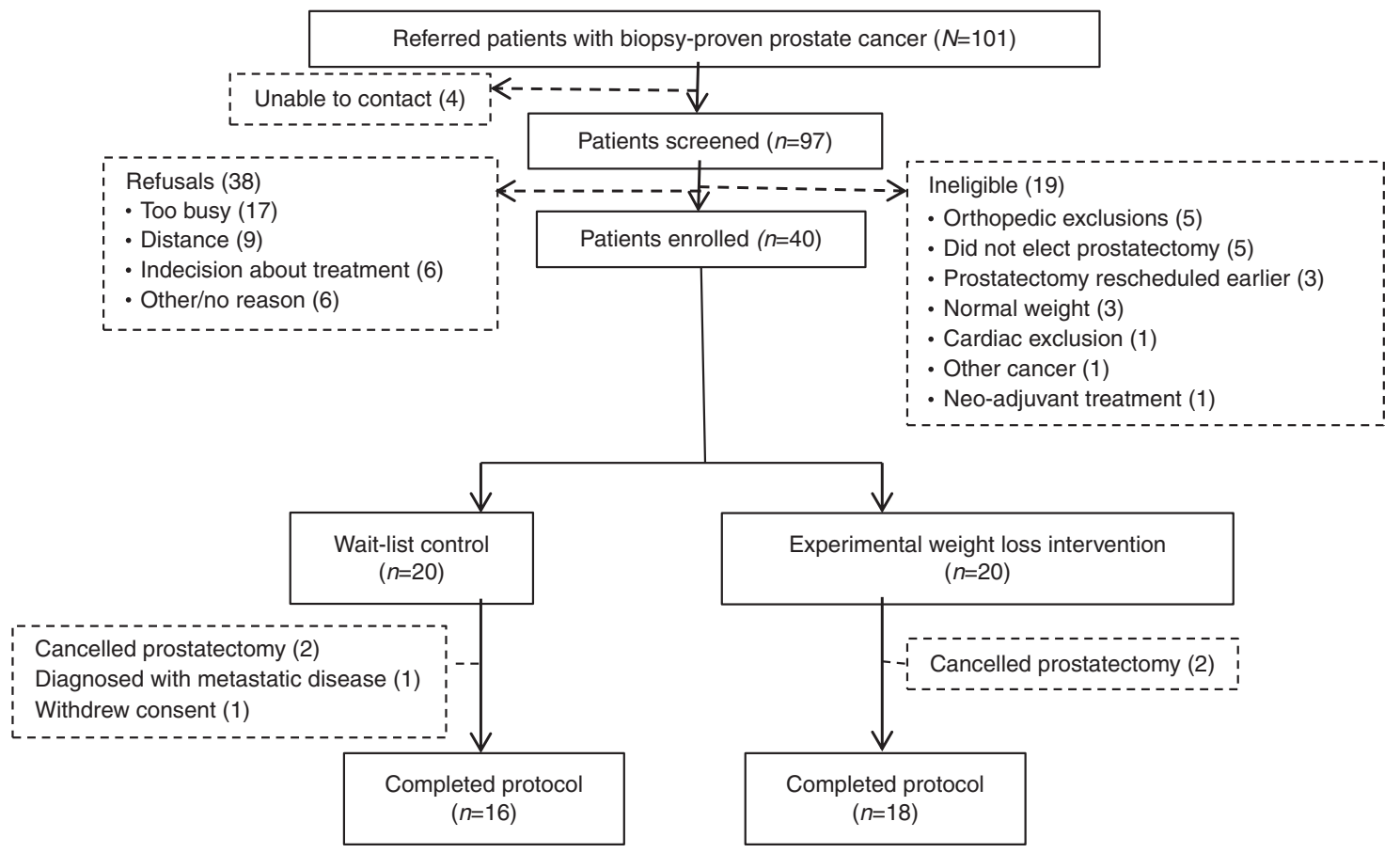

Figure 1. CONSORT diagram. 
Table 1. Baseline characteristics of participants

\begin{tabular}{|c|c|c|c|}
\hline Characteristic & $\begin{array}{c}\text { Total } \\
(n=40)\end{array}$ & $\begin{array}{l}\text { Wait-list } \\
\text { control } \\
(n=20)\end{array}$ & $\begin{array}{l}\text { Weight loss } \\
(n=20)\end{array}$ \\
\hline $\begin{array}{l}\text { Age (years) } \\
\text { Mean (s.d.) } \\
\text { Range }\end{array}$ & $\begin{array}{c}60.1(6.3) \\
46-73\end{array}$ & $\begin{array}{c}60.2(5.9) \\
51-73\end{array}$ & $\begin{array}{c}59.9(6.9) \\
46-72\end{array}$ \\
\hline $\begin{array}{l}\text { Race }(n(\%)) \\
\text { African American } \\
\text { Non-Hispanic White }\end{array}$ & $\begin{array}{l}12(30) \\
28(70)\end{array}$ & $\begin{array}{r}6(30) \\
14(70)\end{array}$ & $\begin{array}{r}6(30) \\
14(70)\end{array}$ \\
\hline $\begin{array}{l}\text { Education ( }(\%)) \\
\text { High school graduate } \\
\text { Some college/technical } \\
\text { College graduate } \\
\text { Post graduate }\end{array}$ & $\begin{array}{r}8(20) \\
12(30) \\
8(20) \\
12(30)\end{array}$ & $\begin{array}{l}5(25) \\
5(25) \\
4(20) \\
6(30)\end{array}$ & $\begin{array}{l}3(15) \\
7(35) \\
4(20) \\
6(30)\end{array}$ \\
\hline $\begin{array}{l}\text { Comorbidities (n (\%)) } \\
0 \\
1-2 \\
3+\end{array}$ & $\begin{array}{c}3(7.9) \\
12(31.6) \\
23(60.5)\end{array}$ & $\begin{array}{c}1(5.3) \\
5(26.3) \\
13(64.4)\end{array}$ & $\begin{aligned} 2 & (10.5) \\
7 & (36.8) \\
10 & (52.7)\end{aligned}$ \\
\hline Cardiovascular disease $(n(\%))$ & $21(53.9)$ & $13(68.4)$ & $8(40.0)$ \\
\hline Diabetes $(n(\%))$ & $10(25)$ & $4(20)$ & $6(30)$ \\
\hline Current smoker (n (\%)) & $5(12.5)$ & $2(10)$ & $3(15)$ \\
\hline $\begin{array}{l}\text { International Society of Urological } \\
\text { Pathology prostate cancer grade } \\
\text { of biopsy ( }(\%)) \\
\text { Grade Group } 1 \text { (Gleason sum } \\
\leqslant 6 \text { ) } \\
\text { Grade Group } 2(3,4) \\
\text { Grade Group } 3(4,3) \\
\text { Grade Group } 4(\text { Gleason sum } 8)\end{array}$ & $\begin{array}{r}11(27.5) \\
16(40.0) \\
8(20.0) \\
5(12.5)\end{array}$ & $\begin{array}{l}5(25.0) \\
7(35.0) \\
5(25.0) \\
3(15.0)\end{array}$ & $\begin{array}{l}6(30.0) \\
9(45.0) \\
3(15.0) \\
2(10.0)\end{array}$ \\
\hline $\begin{array}{l}\text { Body mass index } \\
\text { Mean (s.d.) } \\
\text { Range } \\
\text { Overweight }(n(\%)) \\
\text { Obese }(n(\%))\end{array}$ & $\begin{array}{l}31.4(4.5) \\
25.2-45.8 \\
18(45) \\
22(55)\end{array}$ & $\begin{array}{l}31.8(5.1) \\
25.4-45.8 \\
8(40) \\
12(60)\end{array}$ & $\begin{array}{c}30.9(3.9) \\
25.2-40.3 \\
10(50) \\
10(50)\end{array}$ \\
\hline $\begin{array}{l}\text { Days on protocol } \\
\text { Mean (s.d.) } \\
\text { Range }\end{array}$ & $\begin{array}{c}49.6(23.1) \\
17-95\end{array}$ & $\begin{array}{c}52.2(24.0) \\
17-94\end{array}$ & $\begin{array}{c}47.0(22.6) \\
20-95\end{array}$ \\
\hline
\end{tabular}

Hours of PA was negligible. Objectively measured PA showed slight decreases in both groups. However, no between-arm differences were seen for either of these measures or for fitness.

Little change was noted over time in most physical or emotional quality-of-life subscales or summary scores. However, compared with controls, the WLI arm reported significant improvements in vitality and erection frequency.

Significant between-arm differences were noted in total testosterone and SHBG, with both biomarkers showing strong increases in the WLI arm as compared with controls (Table 3). These concomitant increases of the hormone and its binding protein balanced out the net effect on free androgen index, which remained stable. The WLI arm also experienced significant reductions in leptin as compared with controls. No other between-arm differences were observed with respect to other circulating biomarkers. There also were no significant betweenarm differences in corresponding gene expression within the buffy coat.

Sufficient tissue was secured on $68 \%$ of biopsies and $100 \%$ of surgical specimens. Table 4 chronicles the immunohistochemical results in both the tumour and benign tissue, and within biopsy and surgical specimens. Compared with controls, the WLI arm manifested significantly greater Ki67 proliferation rates at the time of surgery. Change in tumour Ki67 from biopsy to surgery for individual participants is plotted in Figure 2 by study arm, with data provided in Supplementary Table 1. No other between-arm differences were noted in other tumour markers in either the benign or malignant tissue. No significant associations were found between circulating levels of testosterone, SHBG, or leptin, and proliferation rates in the tumour.

The heat map for the top 20 genes corresponding to the signalling and immune pathways that distinguished tumours from the WLI $v s$ control arms shows significant between-arm differences in 13 genes related to signalling and 8 genes related to immune function (Figure 3). The signalling panel detected significant downregulation of serine/threonine kinase 2 (35\% reduction; $P=0.0375)$ and Ephrin A5 (EFNA5; 48\% reduction; $P=0.0098)$, and upregulation of the following genes: AT-rich interaction domain 1A (ARID1A; 14\% increase; $P=0.0330)$; mediator of DNA damage checkpoint 1 ( $M D C 1 ; 14 \%$ increase; $P=0.0078)$; SWI/ SNF-related matrix-associated actin-dependent regulator of chromatin (SMARCA4; 19\% increase; $P=0.0276)$; PHD finger protein 6 (PHF6; 22\% increase; $P=0.0199$ ); glycogen synthase kinase $3 \beta$ (GSK3B; 23\% increase; $P=0.0144$ ); mediator of RNA polymerase II transcription subunit $12(M E D 12 ; 27 \%$ increase; $P=0.04547)$; BCL6 corepressor $(B C O R$; $35 \%$ increase; $P=0.0360)$; activin A receptor $1 \mathrm{~B}(A C V R 1 B$; $36 \%$ increase; $P=0.0475)$; Cal protooncogene $\mathrm{C}(C B L C ; 51 \%$ increase; $P=0.0290)$; DNA polymerase $\beta$ $(P O L B ; 61 \%$ increase; $P=0.0255)$; and Laminin subunit $\gamma-2$ (LAMC2; $207 \%$ increase; $P=0.0480$ ). In addition, the immune panel detected significant downregulation of musculoaponeurotic fibrosarcoma (MAF; 35\% decrease; $P=0.0347$ ) and upregulation of the following genes: signal transducer and activator of transcription 5 (STAT 5B; 14\% increase; $P=0.0403)$; activating transcription factor 1 ( $A T F 1 ; 23 \%$ increase; $P=0.0168)$; Cathepsin L (CTSL; $29 \%$ increase; $P=0.0497)$; transcription factor EB (TFEB; 39\% increase; $P=0.0403$ ); histocompatibility complex, class II, DP Beta 1 (HLA-DPB1; $40 \%$ increase; $P=0.0305)$; mannose receptor $\mathrm{C}$ type $1 \quad(M R C 1 ; 40 \%$ increase; $P=0.0134)$; and cluster of differentiation 86 (CD86; 66\% increase; $P=0.0283$ ).

\section{DISCUSSION}

This trial is the first to document the effects of a presurgical WLI on tumour proliferation rates and other outcomes in patients with cancer. However, instead of reduced tumour proliferation rates as hypothesised, greater proliferation rates were observed with the WLI. As this is the first weight loss trial in the presurgical setting, it is difficult to draw comparisons with other studies, although we had forecasted effects similar to Fabian et al (2013). In that singlearm weight loss study, a median weight loss of $11 \%$ was observed over a 6-month period and Ki67 decreased from 1.4 to $0.4 \%$ $(P=0.041)$ in the subset of women who had detectable proliferation rates in fine-needle periareolar aspirates. However, the Fabian trial differed from the current trial. First, it was performed in women and specifically in healthy women without cancer. Second, the rate of weight loss (\%body weight loss/day) was $64 \%$ faster in the current presurgical trial. Given that Ki67 is expressed more in malignant $v s$ benign tissue and given the steeper trajectory of weight loss in the current trial, we expected an accentuated effect, not one in the opposite direction. This calls into question whether negative energy balance exerts differential effects in transformed cells compared with normal cells. Support for this premise is provided by our data that show no between-arm differences in benign tissue.

Our findings in malignant tissue correspond more to those of Kristal et al (2005), who found increases in Ki67 at 18-month follow-up in the weight loss arm of an RCT of 87 Barrett's oesophagus patients compared with reduced rates among controls. Our findings also parallel those of a recent presurgical trial of metformin among 200 breast cancer patients by Bonanni et al 
Table 2. Baseline to follow-up change in weight status, body composition, diet, physical activity, fitness and quality-of-life

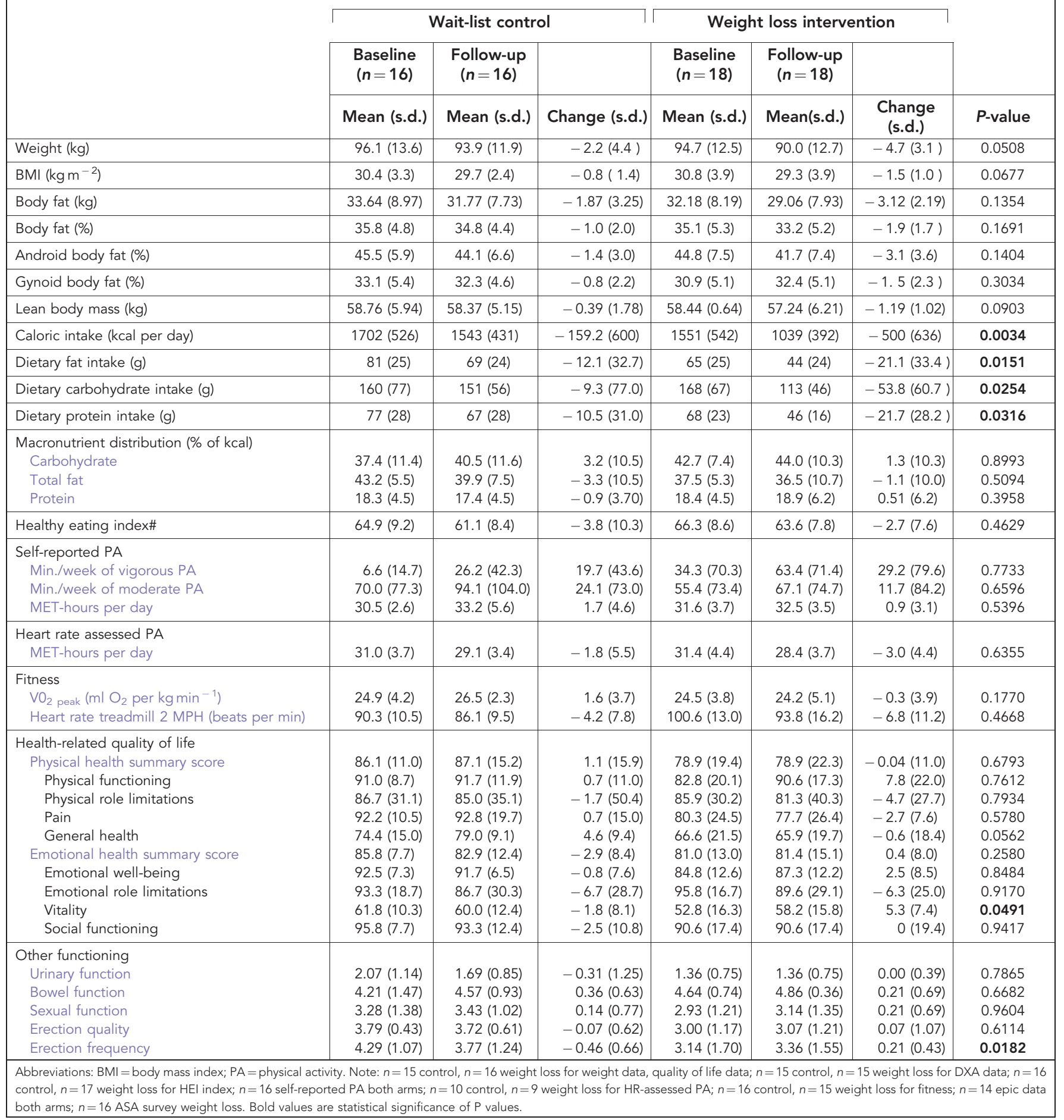

(2012) who observed nonsignificant increases in Ki67 in the metformin arm, who experienced modest weight loss.

Tumour gene expression data, however, support some of our expectations. For example, we found tumour $M A F$ downregulation in WLI men; thus, corroborating findings of Sharad et al (2011) who reported MAF upregulation in tumours of men with high vs low BMIs. Likewise, we expected and found downregulation of EFNA5, a gene associated with insulin secretion; however, we did not see between-arm differences in circulating insulin levels, likely due to the pharmacologic treatment of roughly one-fourth of our sample, who reported a diagnosis of diabetes.

As obesity is associated with decreased immune status (ValdesRamos and Benitez-Arciniega, 2007), we hypothesised upregulation of genes associated with immune response among men who lost weight. Our data support this premise, as $M R C 1, H L A-P B 1$, and CD86 are all indicators of dendritic cell maturation, the cells most able to stimulate T-cell immunity (Karthaus et al, 2012). These data also provide an alternate explanation for the elevated 
Table 3. Baseline to follow-up change in circulating biomarkers

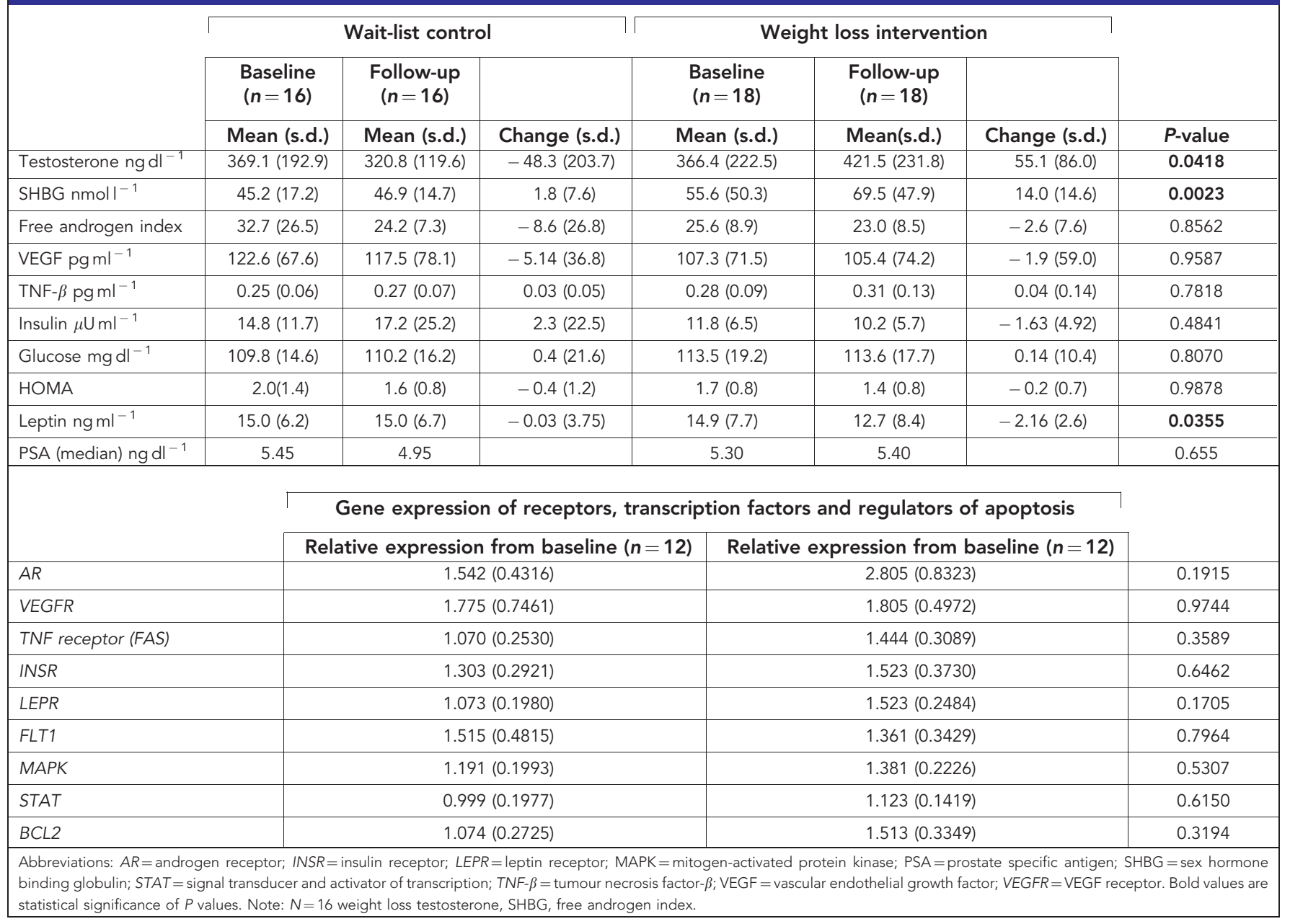

Ki67 detected within tumours in the WLI group: increased intratumoural immune activation following weight loss could contribute to the cellular proliferation detected by Ki67 staining. This hypothesis also is consonant with evidence that weight loss liberates free fatty acids from adipose stores and can trigger inflammation (Calder, 2015), whereas the influx of free fatty acids is generally transitory and the body quickly utilises them for fuel, in the case of more rapid weight loss and little increase in PA to 'burn-off the residual, there may be greater potential for inflammation.

We also hypothesised the upregulation of DNA repair and indeed the increased gene expression of $P O L B$ and $M D C 1$ in the WLI arm bear this out (Wang et al, 2015). A functional coactivator of AR, MDC1 influences cis-regulatory activity of AR target genes via histone $\mathrm{H} 3$ acetylation and, when knocked down, has been shown to increase the growth and migration of both CWR22Rv1 and LNCaP cells (Wang et al, 2015). Thus, within tumours of men losing more weight, we found evidence of favourable immune and insulin regulation, as well as DNA repair.

In contrast, our results also showed upregulation of genes associated with increased transcription, proliferation, migration, and invasion, for example, STAT5B, ATF1, TFEB, PHF6, ACVR1B, $M E D 12, G S K 3 B$, and $L A M C 2$, with increases ranging from $14 \%$ (STAT5B) to $207 \%$ (LAMC2). GSK3B, MED12, and LAMC2 have been suggested as therapeutic targets because of their association with the Wnt signalling/B-catenin pathway, effects on AR, PI3K, and AKT, and contribution to more aggressive prostate cancer (Edwards et al, 2003; Salas et al, 2004; Barbieri et al, 2012).
Therefore, their upregulation with weight loss is unsettling, but may explain the increased Ki67 observed in the WLI vs control arms. Another upregulated gene of interest is $A C V R 1 B$ given its association with androgens, immunosuppression, and carcinogenesis (Nomura et al, 2013).

In men assigned to the WLI, CTSL also was upregulated. This gene is associated with protein catabolism and may be driven by losses in lean mass concomitant with weight loss (Parr et al, 2013). Similar to most weight loss trials, we also detected a solid correlation between the rate of weight loss and the loss of lean mass, although it is interesting to see that weight loss invokes similar catabolic gene expression profiles within the prostatic tumour, as observed in heart or skeletal muscle. Upregulation of CTSL is of concern because of its association with increased osteoclast formation, bone resorption, bone loss, and metastatic potential (Sudhan et al, 2016). Given that the intervention was effective in reducing caloric intake, but not as effective in increasing PA, it is speculated that perhaps the losses in lean mass could have been mitigated and the upregulation of CTSL diminished if we had been able to activate men in the WLI arm, and include both aerobic and resistance PA.

Regarding the host environment, it is clear that the WLI significantly increased both total testosterone and its binding protein (SHBG), thus corroborating results of previous studies (Allan and McLachlan, 2010). Despite significant changes in circulating biomarkers, none of these differences correlated with tumour Ki67. However, the between-arm differences in erection frequency could be the result of androgen shifts. In addition to sex 
Table 4. Immunohistochemical results on tumour and benign tissue in biopsy and surgical specimens

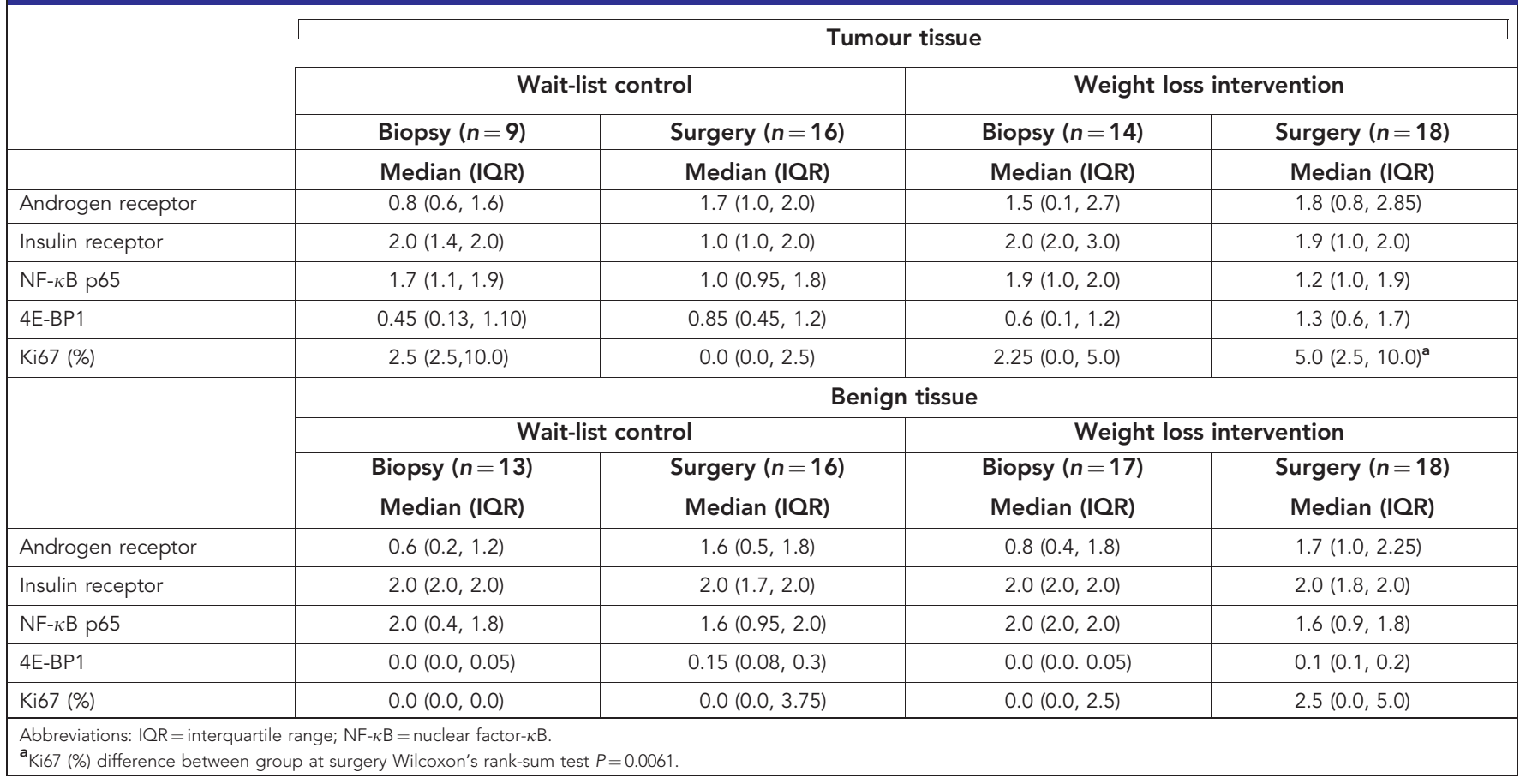
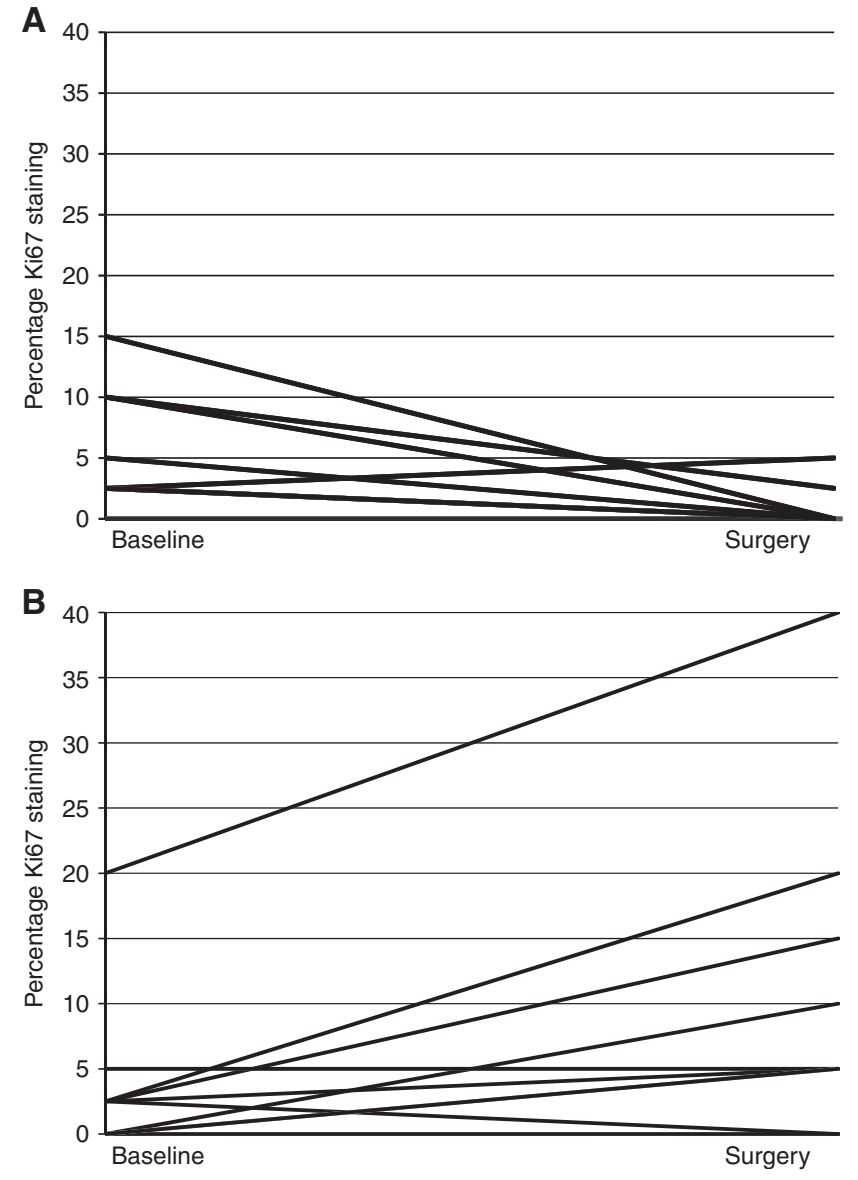

Figure 2. Tumour Ki67 for controls (A) and weight loss group (B) at biopsy and surgery $(P=0.0298$ controls baseline-to-surgery; $P=0.0128$ weight loss group baseline-to-surgery). hormone differences, we, similar to others (Rosenbaum and Leibel, 2014), observed significant decreases in circulating leptin in the WLI vs control arms.

Importantly, significant improvements in vitality were reported by WLI men as compared with controls. Similar between-arm differences were observed in the previously described RENEW trial (Morey et al, 2009). Although other differences in quality-of-life domains may exist, the large variation in response coupled with the small sample size likely precluded our ability to detect differences. Moreover, although the intervention was successful in promoting caloric restriction, it did not result in significant increases in PA, which may have increased quality-of-life to a greater extent (Buffart et al, 2017).

This trial had other limitations besides sample size. Multiple testing increases the risk for false positive findings. Although some correction was used to protect against false discovery in gene expression, this risk still remains for these and other outcomes. Thus, future research is needed to confirm findings of this hypothesis-generating, feasibility trial. A limitation uncommon in pharmacologic trials, but concerning in behavioural interventions, especially among motivated, recently diagnosed patients with cancer, is that of 'drop-in' (Steins Bisschop et al, 2015). Drop-in was a significant issue in this trial, as men in the control group reported caloric reductions, more $\mathrm{PA}$, and lost weight; this drop-in likely attenuated between-arm differences in these factors and others. Prostate cancer also imposes additional limitations: (1) diagnosis from small foci of disease, thus limiting tissue available for pre-post comparisons (adequate tissue was only available on $68 \%$ of biopsy specimens); (2) multi-focality, thus making pre-post comparisons problematic (hence we emphasise between-arm comparisons of surgical specimens, Table 4); and (3) low proliferation rates, increasing the risk of artifact and attenuating the ability to observe differences. Strengths of this presurgical trial are that it is the first ever to assess effects of intentional weight loss on tumours in humans, differences in Ki67 and other outcomes were detected, and the trial met most feasibility benchmarks, that is, retention, adherence, and safety 


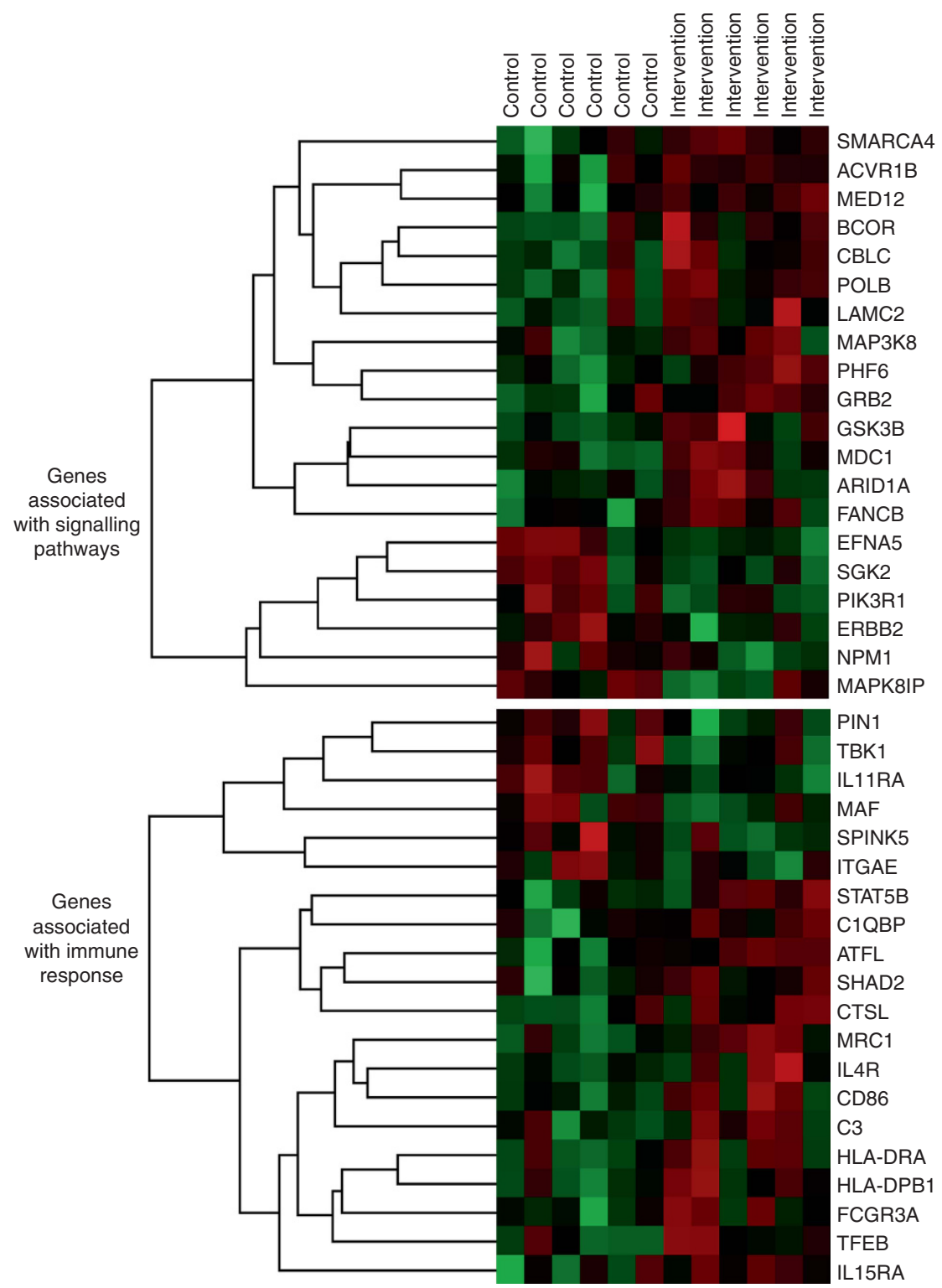

Figure 3. Heat map of the top 20 genes associated with tumour signalling and immune response within surgical tumour specimens of prostate cancer patients assigned to control (left side) vs weight loss (right side) before prostatectomy. Green shading indicates downregulated genes and red shading indicates upregulated genes, with shading intensity indicating departure magnitude from a known standard (black).

(Demark-Wahnefried et al, 2015). Albeit to meet targeted accrual, a 6-month extension was necessary; thus, future trials may require multiple sites, especially if conducted in the US where many surgeries are performed within 3 weeks of diagnosis.

\section{CONCLUSION}

Similar to most feasibility trials, this study raises more questions than it answers, particularly given the unexpected effect on tumour proliferation. Although the results must be cautiously interpreted, they do call for larger studies to replicate findings and answer questions. Specifically, when in the treatment course should weight loss be pursued and how do we optimise dose and content? What is driving increased Ki67 staining, is this associated with a detrimental increase in tumour cell proliferation or a beneficial increase in local immune activation? Some of these concerns are addressed by an American Society of Clinical Oncology report that calls for future research in obesity and cancer (Ligibel et al, 2015).
Until more is known, findings of this study call for caution and support observations by Caan et al (2012) and Lennon et al (2016) who describe an 'obesity paradox in cancer'. Both point to possible dangers of weight loss, with Caan et al (2012) recommending that cancer survivors prevent weight gain, rather than losing weight until more is known, and urging personalised weight management strategies based on medical history. Given the high prevalence of diabetes and cardiovascular disease in our sample, the potential for weight loss to mitigate these competing risks is large and most likely outweighs the threat to cancer control. Clearly, more research is needed to confirm or refute the impact of weight loss, particularly rapid weight loss, on prostate tumour biology.

\section{ACKNOWLEDGEMENTS}

This project is funded by the National Cancer Institute (R21 CA 161263 and P30 CA13148), the National Institute of Diabetes and Digestive and Kidney Diseases (P30 DK079626 and P30 DK56336), and the Webb Family of Birmingham, AL. We acknowledge the 
efforts of the following individuals who contributed their time and expertise to this investigation: Drs Jeffrey Nix, Sunil Sudarshan, and Scott Tully; Tamika Bennett; David Bryan, MA; Deborah Della Manna; Crystal Ellis, RN; Maryellen Williams; Armando Enriquez; Heather Hunter, MS; Brandon L Kane, MA; Margaret Nelson, RN; Denise Oelschager; Scott Tully, Jr; and Amaad Rana. We also are indebted to Elizabeth Platz, ScD, MPH, who reviewed our data and offered suggestions for interpretation. Finally, we are exceptionally grateful to all of the men who participated in this trial and without whom this research would not be possible.

\section{CONFLICT OF INTEREST}

All authors have no conflict of interest, except Drs Soroush Rais Bahrami (Philips/InVivo Corp: Advisor), Eddy S Yang (Bayer, Inc: Advisor/Nanostring Technologies: Honoraria/Lilly, Inc. and Abbvie, Inc. Research Funding), and William E Grizzle (TEVA and Amgen; Stock Ownership/Bristol-Myers Squibb: Advisor, Honoraria and Travel).

\section{REFERENCES}

Allan CA, McLachlan RI (2010) Androgens and obesity. Curr Opin Endocrinol Diabetes Obes 17(3): 224-232.

American Diabetes Association (2007) Choose your foods exchange list diet. Available at: http://glycemic.com/DiabeticExchange/

The\%20Diabetic\%20Exchange\%20List.pdf. Accessed 2015.

Bandura A (2004) Health promotion by social cognitive means. Health Educ Behav 31(2): 143-164.

Barbieri CE, Baca SC, Lawrence MS, Demichelis F, Blattner M, Theurillat JP, White TA, Stojanov P, Van Allen E, Stransky N, Nickerson E, Chae SS, Boysen G, Auclair D, Onofrio RC, Park K, Kitabayashi N, MacDonald TY, Sheikh K, Vuong T, Guiducci C, Cibulskis K, Sivachenko A, Carter SL, Saksena G, Voet D, Hussain WM, Ramos AH, Winckler W, Redman MC, Ardlie K, Tewari AK, Mosquera JM, Rupp N, Wild PJ, Moch H, Morrissey C, Nelson PS, Kantoff PW, Gabriel SB, Golub TR, Meyerson M, Lander ES, Getz G, Rubin MA, Garraway LA (2012) Exome sequencing identifies recurrent SPOP, FOXA1 and MED12 mutations in prostate cancer. Nat Genet 44(6): 685-689.

Benjamini Y, Yekutieli D (2001) The control of the false discovery rate in multiple testing under dependency. Ann Statist 29(4): 1165-1188.

Blair SN, Haskell WL, Ho P, Paffenbarger Jr RS, Vranizan KM, Farquhar JW, Wood PD (1985) Assessment of habitual physical activity by a seven-day recall in a community survey and controlled experiments. Am J Epidemiol 122(5): 794-804.

Bonanni B, Puntoni M, Cazzaniga M, Pruneri G, Serrano D, Guerrieri-Gonzaga A, Gennari A, Trabacca MS, Galimberti V, Veronesi P, Johansson H, Aristarco V, Bassi F, Luini A, Lazzeroni M, Varricchio C, Viale G, Bruzzi P, Decensi A (2012) Dual effect of metformin on breast cancer proliferation in a randomized presurgical trial. J Clin Oncol 30(21): 2593-2600.

Buffart LM, Kalter J, Sweegers MG, Courneya KS, Newton RU, Aaronson NK, Jacobsen PB, May AM, Galvao DA, Chinapaw MJ, Steindorf K, Irwin ML, Stuiver MM, Hayes S, Griffith KA, Lucia A, Mesters I, van Weert E, Knoop H, Goedendorp MM, Mutrie N, Daley AJ, McConnachie A, Bohus M, Thorsen L, Schulz KH, Short CE, James EL, Plotnikoff RC, Arbane G, Schmidt ME, Potthoff K, van Beurden M, Oldenburg HS, Sonke GS, van Harten WH, Garrod R, Schmitz KH, Winters-Stone KM, Velthuis MJ, Taaffe DR, van Mechelen W, Kersten MJ, Nollet F, Wenzel J, Wiskemann J, Verdonck-de Leeuw IM, Brug J (2017) Effects and moderators of exercise on quality of life and physical function in patients with cancer: an individual patient data meta-analysis of 34 RCTs. Cancer Treat Rev 52: 91-104.

Caan BJ, Kwan ML, Shu XO, Pierce JP, Patterson RE, Nechuta SJ, Poole EM, Kroenke CH, Weltzien EK, Flatt SW, Quesenberry Jr CP, Holmes MD, Chen WY (2012) Weight change and survival after breast cancer in the after breast cancer pooling project. Cancer Epidemiol Biomarkers Prev 21(8): 1260-1271
Calder PC (2015) Functional roles of fatty acids and their effects on human health. J Parenteral Enteral Nutr 39(1 Suppl): 18s-32s.

Cao Y, Ma J (2011) Body mass index, prostate cancer-specific mortality, and biochemical recurrence: a systematic review and meta-analysis. Cancer Prev Res (Phila) 4(4): 486-501.

De Keyzer W, Huybrechts I, De Vriendt V, Vandevijvere S, Slimani N, Van Oyen H, De Henauw S (2011) Repeated 24-hour recalls versus dietary records for estimating nutrient intakes in a national food consumption survey. Food Nutr Res 55.

Demark-Wahnefried W, Nix JW, Hunter GR, Rais-Bahrami S, Desmond RA, Chacko B, Morrow CD, Azrad M, Fruge AD, Tsuruta Y, Ptacek T, Tully SA, Segal R, Grizzle WE (2015) Feasibility outcomes of a presurgical randomized controlled trial exploring the impact of caloric restriction and increased physical activity versus a wait-list control on tumor characteristics and circulating biomarkers in men electing prostatectomy for prostate cancer. BMC Cancer 16: 61.

Demark-Wahnefried W, Polascik TJ, George SL, Switzer BR, Madden JF, MTt Ruffin, Snyder DC, Owzar K, Hars V, Albala DM, Walther PJ, Robertson CN, Moul JW, Dunn BK, Brenner D, Minasian L, Stella P, Vollmer RT (2008) Flaxseed supplementation (not dietary fat restriction) reduces prostate cancer proliferation rates in men presurgery. Cancer Epidemiol Biomarkers Prev 17(12): 3577-3587.

Dowsett M, Dunbier AK (2008) Emerging biomarkers and new understanding of traditional markers in personalized therapy for breast cancer. Clin Cancer Res 14(24): 8019-8026.

Edwards J, Krishna NS, Witton CJ, Bartlett JM (2003) Gene amplifications associated with the development of hormone-resistant prostate cancer. Clin Cancer Res 9(14): 5271-5281.

Fabian CJ, Kimler BF, Donnelly JE, Sullivan DK, Klemp JR, Petroff BK, Phillips TA, Metheny T, Aversman S, Yeh HW, Zalles CM, Mills GB, Hursting SD (2013) Favorable modulation of benign breast tissue and serum risk biomarkers is associated with $>10 \%$ weight loss in postmenopausal women. Breast Cancer Res Treat 142(1): 119-132.

Fillenbaum G (1988) Multidimensional Functional Assessment of Older Adults. Lawrence Erlbaum Associates: Hillsdale, NJ.

Frankenfield DC, Rowe WA, Smith JS, Cooney RN (2003) Validation of several established equations for resting metabolic rate in obese and nonobese people. J Am Diet Assoc 103(9): 1152-1159.

Hadziabdic MO, Mucalo I, Hrabac P, Matic T, Rahelic D, Bozikov V (2015) Factors predictive of drop-out and weight loss success in weight management of obese patients. J Hum Nutr Diet 28(Suppl 2): 24-32.

Hays RD, Sherbourne CD, Mazel RM (1993) The RAND 36-Item Health Survey 1.0. Health Econ 2(3): 217-227.

Hursting SD, Digiovanni J, Dannenberg AJ, Azrad M, Leroith D, DemarkWahnefried W, Kakarala M, Brodie A, Berger NA (2012) Obesity, energy balance, and cancer: new opportunities for prevention. Cancer Prev Res (Phila) 5(11): 1260-1272.

Jayachandran J, Banez LL, Aronson WJ, Terris MK, Presti Jr JC, Amling CL, Kane CJ, Freedland SJ (2009) Obesity as a predictor of adverse outcome across black and white race: results from the Shared Equal Access Regional Cancer Hospital (SEARCH) Database. Cancer 115(22): 5263-5271.

Jensen MD, Ryan DH, Apovian CM, Ard JD, Comuzzie AG, Donato KA, $\mathrm{Hu}$ FB, Hubbard VS, Jakicic JM, Kushner RF, Loria CM, Millen BE, Nonas CA, Pi-Sunyer FX, Stevens J, Stevens VJ, Wadden TA, Wolfe BM, Yanovski SZ (2014) 2013 AHA/ACC/TOS guideline for the management of overweight and obesity in adults: a report of the American College of Cardiology/American Heart Association Task Force on Practice Guidelines and The Obesity Society. J Am Coll Cardiol 63(25 Pt B): 2985-3023.

Kachroo N, Gnanapragasam VJ (2013) The role of treatment modality on the utility of predictive tissue biomarkers in clinical prostate cancer: a systematic review. J Cancer Res Clin Oncol 139(1): 1-24.

Karthaus N, Torensma R, Tel J (2012) Deciphering the message broadcast by tumor-infiltrating dendritic cells. Am J Clin Pathol 181(3): 733-742.

Kelloff GJ, Boone CW, Steele VE, Crowell JA, Lubet R, Sigman CC (1994) Progress in cancer chemoprevention: perspectives on agent selection and short-term clinical intervention trials. Cancer Res 54(7 Suppl): 2015s-2024s.

Kohl HW, Gibbons LW, Gordon NF, Blair SN (1990) An empirical evaluation of the ACSM guidelines for exercise testing. Med Sci Sports Exerc 22(4): 533-539.

Kristal AR, Blount PL, Schenk JM, Sanchez CA, Rabinovitch PS, Odze RD, Standley J, Vaughan TL, Reid BJ (2005) Low-fat, high fruit and vegetable 
diets and weight loss do not affect biomarkers of cellular proliferation in Barrett esophagus. Cancer Epidemiol Biomarkers Prev 14(10): 2377-2383.

Lauby-Secretan B, Scoccianti C, Loomis D, Grosse Y, Bianchini F, Straif K (2016) Body fatness and cancer-viewpoint of the IARC Working Group. N Engl J Med 375(8): 794-798.

Lennon H, Sperrin M, Badrick E, Renehan AG (2016) The obesity paradox in cancer: a review. Curr Oncol Rep 18(9): 56.

Ligibel JA, Alfano CM, Hershman D, Ballard RM, Bruinooge SS, Courneya KS, Daniels EC, Demark-Wahnefried W, Frank ES, Goodwin PJ, Irwin ML, Levit LA, McCaskill-Stevens W, Minasian LM, O'Rourke MA, Pierce JP, Stein KD, Thomson CA, Hudis CA (2015) Recommendations for obesity clinical trials in cancer survivors: American Society of Clinical Oncology statement. J Clin Oncol 33(33): 3961-3967.

Litwin MS, Hays RD, Fink A, Ganz PA, Leake B, Brook RH (1998) The UCLA Prostate Cancer Index: development, reliability, and validity of a healthrelated quality of life measure. Med Care 36(7): 1002-1012.

Morey MC, Snyder DC, Sloane R, Cohen HJ, Peterson B, Hartman TJ, Miller P, Mitchell DC, Demark-Wahnefried W (2009) Effects of home-based diet and exercise on functional outcomes among older, overweight long-term cancer survivors: RENEW: a randomized controlled trial. J Am Med Assoc 301(18): 1883-1891.

Nomura M, Tanaka K, Wang L, Goto Y, Mukasa C, Ashida K, Takayanagi R (2013) Activin type IB receptor signaling in prostate cancer cells promotes lymph node metastasis in a xenograft model. Biochem Biophys Res Commun 430(1): 340-346.

Parr EB, Coffey VG, Hawley JA (2013) 'Sarcobesity': a metabolic conundrum. Maturitas 74(2): 109-113.

Reeves MM, Terranova CO, Eakin EG, Demark-Wahnefried W (2014) Weight loss intervention trials in women with breast cancer: a systematic review. Obes Rev 15(9): 749-768.

Rock CL, Doyle C, Demark-Wahnefried W, Meyerhardt J, Courneya KS, Schwartz AL, Bandera EV, Hamilton KK, Grant B, McCullough M, Byers T, Gansler T (2012) Nutrition and physical activity guidelines for cancer survivors. CA Cancer J Clin 62(4): 243-274.

Rosenbaum M, Leibel RL (2014) 20 years of leptin: role of leptin in energy homeostasis in humans. J Endocrinol 223(1): T83-T96.

Salas TR, Kim J, Vakar-Lopez F, Sabichi AL, Troncoso P, Jenster G, Kikuchi A, Chen SY, Shemshedini L, Suraokar M, Logothetis CJ, DiGiovanni J, Lippman SM, Menter DG (2004) Glycogen synthase kinase3 beta is involved in the phosphorylation and suppression of androgen receptor activity. J Biol Chem 279(18): 19191-19200.

Schenk JM, Neuhouser ML, Lin DW, Kristal AR (2009) A dietary intervention to elicit rapid and complex dietary changes for studies investigating the effects of diet on tissues collected during invasive surgical procedures. J Am Diet Assoc 109(3): 459-463.

Schmitz KH, Courneya KS, Matthews C, Demark-Wahnefried W, Galvao DA, Pinto BM, Irwin ML, Wolin KY, Segal RJ, Lucia A, Schneider CM, von Gruenigen VE, Schwartz AL (2010) American College of Sports Medicine roundtable on exercise guidelines for cancer survivors. Med Sci Sports Exerc 42(7): 1409-1426.

Sharad S, Srivastava A, Ravulapalli S, Parker P, Chen Y, Li H, Petrovics G, Dobi A (2011) Prostate cancer gene expression signature of patients with high body mass index. Prostate Cancer Prostatic Dis 14(1): $22-29$.

Steins Bisschop CN, Courneya KS, Velthuis MJ, Monninkhof EM, Jones LW, Friedenreich C, van der Wall E, Peeters PH, May AM (2015) Control group design, contamination and drop-out in exercise oncology trials: a systematic review. PLoS One 10(3): e0120996.

Subar AF, Kirkpatrick SI, Mittl B, Zimmerman TP, Thompson FE, Bingley C, Willis G, Islam NG, Baranowski T, McNutt S, Potischman N (2012) The automated self-administered 24-hour dietary recall (ASA24): a resource for researchers, clinicians and educators from the National Cancer Institute. J Acad Nutr Diet 112(8): 1134-1137.

Sudhan DR, Pampo C, Rice L, Siemann DW (2016) Cathepsin L inactivation leads to multimodal inhibition of prostate cancer cell dissemination in a preclinical bone metastasis model. Int J Cancer 138(11): 2665-2677.

TG Lohman AR, Martorell R (1988) Anthropometric Standardization Reference Manual. Human Kinetics Books: Champaign.

US Bureau of the Census (2014) America Community Survey, 2010-2014. (accessed 22 August 2017). https://www.census.gov/programs-surveys/acs/.

Valdes-Ramos R, Benitez-Arciniega AD (2007) Nutrition and immunity in cancer. Br J Nutr 98(Suppl 1): S127-S132.

Wang C, Sun H, Zou R, Zhou T, Wang S, Sun S, Tong C, Luo H, Li Y, Li Z, Wang E, Chen Y, Cao L, Li F, Zhao Y (2015) MDC1 functionally identified as an androgen receptor co-activator participates in suppression of prostate cancer. Nucleic Acids Res 43(10): 4893-4908.

Wright JL, Plymate S, D’Oria-Cameron A, Bain C, Haugk K, Xiao L, Lin DW, Stanford JL, McTiernan A (2013) A study of caloric restriction versus standard diet in overweight men with newly diagnosed prostate cancer: a randomized controlled trial. Prostate 73(12): 1345-1351.

Yuan C, Cao Y, Chavarro J, Lindstrom S, Qiu W, Willett W, Hsing A, Kibel A, Rosner B, Stampfer M, Kra P, Ma J (2015) Prediagnostic body-mass index, smoking and prostate cancer survival: a Cohort Consortium Study of over 10000 White men with prostate cancer. Cancer Epidemiol Biomarkers Prev 24(4): 759-760.

(c) (1)(2) This work is licensed under the Creative Commons Attribution-Non-Commercial-Share Alike 4.0 International License. To view a copy of this license, visit http:// creativecommons.org/licenses/by-nc-sa/4.0/

(C) The Author(s) named above 2017

Supplementary Information accompanies this paper on British Journal of Cancer website (http://www.nature.com/bjc) 